\title{
Stabilization of the Inertia Wheel Inverted Pendulum by Advanced IDA-PBC Based Controllers: Comparative Study and Real-Time Experiments
}

\author{
Afef Hfaiedh \\ (a) University of Carthage, National Engineering School of Carthage \\ (b) Laboratory of Research in Automatic (LA.R.A) \\ Tunis, Tunisia \\ afef.hfaiedh@enicar.u-carthage.tn
}

\author{
Ahmed Chemori \\ LIRMM, University of Montpellier, CNRS \\ Montpellier, France \\ ahmed.chemori@lirmm.fr
}

\author{
Afef Abdelkrim ${ }^{a, b}$ \\ afef.a.abdelkrim@ieee.org
}

\begin{abstract}
Interconnection and Damping Assignment Passivity Based Control (IDA-PBC) is a popular control scheme, for the stabilization of underactuated mechanical systems, formulated in Port-Controlled Hamiltonian (PCH) structure. However, robustness enhancement of this control approach towards external disturbances remains an challenging and an open problem. In this paper, an experimental comparative study between two different IDAPBC approaches is proposed. The first controller is a nonlinear Proportional Integral IDA-PBC, while the second one is a model reference adaptive IDA-PBC. To evaluate the effectiveness of both controllers, various real-time experimental scenarios have been conducted for the stabilization of the inertia wheel inverted pendulum. For the sake of a fair comparison, different performance-evaluation criteria have been proposed to quantify the control performance in terms of convergence and energy consumption. The results show a better performance of the nonlinear Proportional Integral IDA-PBC controller compared to the model reference adaptive IDA-PBC controller.
\end{abstract}

Index Terms-Underactuated mechanical system, stabilization, IDA-PBC controller, adaptive control.

\section{Definition AND Preliminaries}

- We denote an $n \times n$ identity matrix by $I_{n}$.

- The set of real numbers (including 0 ) is denoted $\mathbb{R}$.

- Given an arbitrary matrix $G$, its transpose is denoted by $G^{T}$.

- $G^{\perp}$ denotes the full rank left annihilator of $\mathrm{G}$ where $G^{\perp} G=0$.

- For a vector $x \in \mathbb{R}^{n}$, a matrix $A \in \mathbb{R}^{n \times n}$, with $A=$ $A^{T}>0,|x|$ is the Euclidean norm as $|x|^{2}=x^{T} x$ and the weighted norm is denoted by $\|x\|_{A}^{2}=x^{T} A x$.

- Given a continuous function $H(i, j): \mathbb{R}^{n} \rightarrow \mathbb{R}$, the gradient is denoted by $\nabla_{i} H:=\left(\frac{\partial H}{\partial i}\right)^{T}$.

\section{INTRODUCTION}

Control of underactuated mechanical systems (UMSs) is an interesting area of research. Underactuation can be decided intentionally to decrease the weight and reduce the cost of design [1]. Furthermore, the control of such systems may represent a viable control solution in case of an actuator failure. It would guarantee the good operation of the system and ensures the continuity of difficult missions [2]. However, this feature presents a significant challenge in terms of control point of view, compared to the case of fully actuated systems. Indeed, underactuated systems with fewer control inputs than degrees of freedom, are generally nonlinear, often characterized by a non-minimum phase behaviour and the Lagrangian dynamics may contain nonholonomic constraints [3] [4]. For these reasons, the control design leads to complex theoretical problems that cannot be solved using classical control techniques.

To deal with this issue some researchers refer to representing the system in Port-Controlled Hamiltonian $(\mathrm{PCH})$ formulation. Writing a system in a $\mathrm{PCH}$ form [5] has the advantage of covering a large set of physical systems and providing important structural properties [6]. It provides the relation between the dynamics and the energy of the system, the coupling between the non-damping and damping elements [7]. Another formulation of passivity based control (PBC), namely the Interconnection and Damping Assignment-Passivity Based Control (IDA$\mathrm{PBC}$ ) was proposed in [8] to control underactuated $\mathrm{PCH}$ systems. It is a successful control approach in the context of mechanical systems presented through Euler-Lagrange formulation. It has been used to control various systems, e.g. Ball and beam [8], inertia wheel inverted pendulum [8], power converters [9], induction motor [10], non minimum phase chemical reactor [11], flexible Spacecraft [12], among others. It invokes the principle of energy shaping and dissipation, where the closed-loop energy function may obtained through the solution of partial 
differential equations (PDEs) called matching equations.

Many theoretical extensions and practical applications of this approach have already been reported in literature. For instance, a theoretical analysis about the presence of physical damping in open-loop hamiltonian formulation was conducted in [13]. Two methods namely passivation by interconnection assignment and passivation by damping injection were given for the control redesign. These methods were tested in simulation on the ball and beam system with Coulomb friction and on the Vertical Takeoff and Landing (VTOL) aircraft.

It is worth to note that, it is a hard task to resolve PDEs to compute the kinetic and potential energy functions. Transforming the set of PDEs to a set of ODEs is a method presented in [14] in order to simplify the resolution of the partial differential equations associated to the kinetic energy. This approach is based on the re-parametrization of the target dynamics and has been applied to the cart and Furuta pendulums. Via a parametrization of the closed-loop inertia matrix, another simplification of the PDE equation, associated to the potential energy was proposed in [15] and has been applied to solve a global stabilization design of a rotary inverted pendulum.

Based on IDA-PBC scheme, several control approaches were reported in the literature. Taking care of the actuator torque restriction and to ensure a bounded control action, a modification of IDA-PBC controller was proposed in [16] by inclusion of a tangent function in the control law. A discrete-time design of IDA-PBC approach was proposed in [17] for separable Hamiltonian dynamics (i.e Inertia matrix is constant). This approach was tested for the stabilization of the Inertia Wheel Inverted Pendulum (IWIP) for different values of the sampling period.

To deal with the robustness issue of IDA-PBC controller, many solutions were investigated in literature. The inclusion of Integral control (IC) and PID control have recently reported in several works. For instance, in [18] a PID-like controller was proposed for the robustification problem of separable PCH systems. Assuming that the stabilization problem is solved by IDAPBC method, the authors introduced an integral action to solve the robustness issue of fully actuated system. This approach was extended to control underactuated mechanical systems where the (IC) was included on the non-passive output. The main idea was reported in [19], it consists in adding an integral action on the non-passive outputs to deal with the problem of constant and matched disturbances rejection in underactuated mechanical systems with validation through real-time experiments. A Model Reference Adaptive Control (MRAC) combined with IDA-PBC controller was proposed in [20] [6]. The proposed approach compensates the external disturbances better than the standard IDA-PBC proposed in [8]. The efficiency of this approach was demonstrated throught simulation and real-time experiments for the stabilization of the Inertia Wheel Inverted Pendulum (IWIP) subject to matched and unmatched disturbances.

In this paper, we propose a comparative study of two robust approaches, the nonlinear PI controller developed in [19] and the Model Reference Adaptive control proposed in [6]. It is worth to note that these controllers were successfully compared in experiments with respect to the standard version of IDA-PBC [8].

The main contribution of this work is a comparative study between two IDA-PBC based controllers. The first is the PI-IDA-PBC [19] and the second one is the MRA-IDA-PBC [6]. The study is based on some known performance-evaluation criteria used to quantify the performance of both controllers in terms of stabilization and energy consumption. The main focuses of this paper is that the comparative analysis is done by real-time experiments. Validation is performed in a real underactuated mechanical system, namely the inertia wheel inverted pendulum. As a second contribution, different scenarios were considered in our comparative study, in order to analyse the performance of both controllers.

The rest of this paper is organized as follows. Section III presents a briefly review on the two implemented controllers. Section IV presents the application on the Inertia Wheel Inverted Pendulum. Section V is devoted to the experimental results. Conclusion and future work are addressed in section VI.

\section{BACKGROUND ON IDA-PBC BASED CONTROLLERS}

In this section, we consider two advanced control schemes based on IDA-PBC, proposed in [19] [6]. The standard IDA-PBC [8] is a well known control method used for the stabilization of various underactuated mechanical systems. Based on Hamiltonian formalism, this technique is robust against parameter uncertainties. It combines the passivity properties of (Port-Controlled Hamiltonian systems) PCHs and control by interconnection and energy based control [8]. The first method is the PI-IDA-PBC, it consists in a first extension by adding an outer-loop PI controller to the original IDA-PBC in order to improve its robustness [19]. The second one is an improvement of the IDA-PBC controller by an adaptation term in order to compensate the errors on the uncertain parameters [6] [21].

\section{A. Standard IDA-PBC controller}

The equations of motion of an underactuated mechanical system can be written in Port-Controlled Hamiltonian form $(\mathrm{PCH})$ as

$$
\begin{gathered}
{\left[\begin{array}{c}
\dot{q} \\
\dot{p}
\end{array}\right]=\left[\begin{array}{cc}
0_{n \times n} & I_{n} \\
I_{n} & 0_{n \times n}
\end{array}\right] \nabla H(q, p)+\left[\begin{array}{c}
0_{n \times m} \\
G(q)
\end{array}\right] u(1)} \\
y=G(q)^{T} \nabla_{p} H
\end{gathered}
$$

where $(q, p) \in \mathbb{R}^{n}$ are respectively the generalized position and momenta, $u \in \mathbb{R}^{m}$ is the control input vector, 
$G(q): \mathbb{R}^{n} \rightarrow \mathbb{R}^{n \times m}$ is the input matrix with $\operatorname{rank}(G)=$ $m<n, y$ is the output vector and $H: \mathbb{R}^{n} \times \mathbb{R}^{n} \rightarrow \mathbb{R}$ is the total energy. This last one is defined as the sum of the kinetic and potential energy, expressed as follows

$$
H(q, p):=\frac{1}{2} p^{T} M^{-1}(q) p+V(q)
$$

where $M: \mathbb{R}^{n} \rightarrow \mathbb{R}^{n \times n}$ is a positive definite inertia matrix, $M(q)=M(q)^{T}>0$ and $V(q): \mathbb{R}^{n} \rightarrow \mathbb{R}$ is the potential energy. The IDA-PBC control law is expressed as the sum of two terms, namely

$$
u_{i d a}=u_{e s}(q, p)+u_{d i}(q, p)
$$

where the first one is the energy shaping control to assign the desired equilibrium $\left(q^{*}, 0\right)$, found by solving a set of PDEs equations while the closed-loop system is lossless [15], for more details the reader can refer to [8].

$$
u_{e s}=\left(G^{T} G\right)^{-1} G^{T}\left(\nabla_{q} H-M_{d} M^{-1} \nabla_{q} H_{d}+J_{2} M_{d}^{-1} p\right)
$$

where $M_{d} \in \mathbb{R}^{n \times m}$ is a positive definite desired inertia matrix, $H_{d} \in \mathbb{R}^{n \times n}$ is the desired energy expressed as $H_{d}(q, p)=\frac{1}{2} p^{T} M_{d}(q)^{-1} p+V_{d}(q)$ and $V_{d}(q)$ is the desired potential energy verifying the condition to have an isolated minimum at $q^{*}$ defined by

$$
q^{*}=\arg \min \left(V_{d}\right)
$$

$J_{2}$ is a free tuned parameter which fulfills the skewsymmetry condition, that is

$$
J_{2}(q, p)=-J_{2}^{T}(q, p)
$$

The second term of the control input injects damping to achieve asymptotic stability via a negative feedback of the passive output. It is expressed as

$$
u_{d i}=\left(-K_{v}\right) G^{T} \nabla_{p} H_{d}
$$

where $u_{d i} \in \mathbb{R}^{m}$ and $K_{v}=K_{v}^{T}>0$. The desired (closedloop) $\mathrm{PCH}$ dynamics has a desired equilibrium point at $\left(q^{*}, 0\right)$. It is expressed as

$$
\left(\begin{array}{c}
\dot{q} \\
\dot{p}
\end{array}\right)=\left(J_{d}(q, p)-R_{d}(q, p)\right)\left(\begin{array}{c}
\nabla_{q} H_{d} \\
\nabla_{p} H_{d}
\end{array}\right)
$$

where $J_{d}(q, p)=-J_{d}^{T}(q, p)$ is the interconnection matrix and $R_{d}=R_{d}^{T}$ is the damping matrix. They are respectively expressed by

$$
\begin{gathered}
J_{d}=\left(\begin{array}{cc}
0 & M^{-1} M_{d} \\
-M_{d} M^{-1} & J_{2}
\end{array}\right) \\
R_{d}=\left(\begin{array}{cc}
0 & 0 \\
0 & G K_{\nu} G^{T}
\end{array}\right)
\end{gathered}
$$

The closed-loop system in Eq.(9) has a stable equilibrium point at $\left(q^{*}, 0\right)$ with the Lyapunov function

$$
H_{d}(q, p):=\frac{1}{2} p^{T} M_{d}^{-1}(q) p+V_{d}(q)
$$

where $V_{d}$ is the desired potential energy verify the condition (6). The first derivative of (12) is expressed by

$$
\dot{H}_{d}(q, p)=-\left\|G^{T} M_{d}^{-1} p\right\|_{k_{p}}^{2} \leq 0
$$

\section{B. PI-IDA-PBC controller}

The nonlinear PI controller consists in an outer-loop controller designed in order to solve the problem of constant disturbance rejection for underactuated mechanical systems. In accordance with the first proposition (Eq.(9) and Eq.(10)) in [19], the control input is defined as the sum of the standard IDA-PBC controller Eq.(4) and mathematical expression of PI controller $\beta(q, \zeta)$.

$$
u_{p i}=u_{i d a}+\beta(q, \zeta)
$$

The objective is to design a controller $\beta(q, \zeta)$ in order to ensure asymptotic stability of the desired equilibrium $\left(q^{*}, \zeta^{*}\right)$.

$$
\beta(q, \zeta)=-K_{2} K_{I} K_{2}^{T} G^{T} M^{-1} \nabla V_{d}-K_{p} K_{I} \zeta
$$

where $K_{p}>0, K_{I}>0$ are constant matrices. The dynamics of the controller state $\zeta \in \mathbb{R}^{n}$ is expressed as follows

$$
\dot{\zeta}=K_{2}^{T} G^{T} M^{-1} \nabla V_{d}
$$

The expression of the matrix $K_{2}$ includes the input matrix $G$ and the desired inertia matrix $M_{d}$,

$$
K_{2}=\left(G^{T} M_{d}^{-1} G\right)^{-1}
$$

This approach requires some assumptions which are not satisfied for all kind of underactuated mechanical systems. For more details about the proof of stability, the reader can refer to [19].

\section{MRA-IDA-PBC controller}

Considering that the gains values of the IDA-PBC control law $u_{i d a}$ are assumed to be uncertain. The MRAIDA-PBC controller is a direct model reference adaptive controller in which the parameters of IDA-PBC controller are estimated online, in order to ensure better performances and to deal with matched disturbances. The control input is given by

$$
u_{M R A}=u_{i d a}+\Delta(x, t) \hat{z}
$$

where $u_{i d a}$ denotes the IDA-PBC control law expressed in Eq.(4), $\Delta(x, t)$ is a matrix of defined functions, $z=$ $\left[z_{1} z_{2} \ldots z_{p}\right]^{T}$ presents the vector of unknown parameters and $\hat{z}$ denotes the estimate of $z$ with the following adaptation law

$$
\hat{\dot{z}}=-K_{a} \Delta(x, t) y
$$

where $K_{a}$ is chosen as a diagonal positive definite matrix. The estimation error is then expressed as

$$
\tilde{z}=\hat{z}-z
$$

The closed-loop system with the error $\tilde{z}$ can be written as follows 


$$
\left.\left[\begin{array}{c}
\dot{q} \\
\dot{p} \\
\dot{\tilde{z}}
\end{array}\right]=\left[\begin{array}{lll}
0 & M^{-1} M_{d} & 0 \\
-M_{d} M^{-1} & J_{2}-G k_{v} G^{T} & G \Delta K_{a} \\
0 & -K_{a} \Delta^{T} G^{T} & 0
\end{array}\right]\left[\begin{array}{l}
\nabla_{q} \bar{H} \\
\nabla_{p} \bar{H}_{22} \\
\nabla_{z} \bar{H}
\end{array}\right]\right)
$$

where the hamiltonian $\bar{H}$ is given by

$$
\bar{H}(q, p, \tilde{z})=H_{d}(q, p)+\frac{1}{2} \tilde{z}^{T} K_{a}^{-1} \tilde{z}
$$

The stability analysis is demonstrated by choosing $\bar{H}(q, p, \tilde{z})$ as a Lyapunov candidate function [20] [6].

$$
\begin{aligned}
& \dot{\bar{H}}=\dot{H}_{d}+K_{a}^{-1} \bar{z} \dot{\bar{z}} \\
& \dot{\bar{H}}=\dot{H}_{d}+K_{a}^{-1} \bar{z} \Delta^{T}(x, t) G^{T} \nabla_{p} H_{d} \\
& \dot{\bar{H}}=\nabla_{q} H_{d} \dot{q}+\nabla_{p} H_{d} \dot{p}+K_{a}^{-1} \bar{z} \Delta^{T}(x, t) G^{T} \nabla_{p} H_{d} \\
& \dot{\bar{H}} \leq-\alpha\left|\nabla_{p} H_{d}^{T} G\right|^{2}
\end{aligned}
$$

where $\alpha$ is a positive constant.

\section{Application: THE INERTIA WHEEL INVERTED PENDULUM (IWIP)}

The inertia wheel inverted pendulum is a well known nonlinear underactuated mechanical system [22]. It had attracted the attention of many researchers within the control community and was considered as a benchmark experiment to study new nonlinear control methodologies [23] [24] [25]. In this section, we present the application of the two approaches based on IDA-PBC described in the previous section.

\section{A. Description and model transformation}

The inertia wheel inverted pendulum is an underactuated mechanical system [26] with two degrees of freedom and one control input like the rotary inverted pendulum [27] and cart-pole pendulum [28]. The pendulum angle $\theta_{1}$ with respect to vertical axis is unactuated. The joint between the body and the inertia wheel $\theta_{2}$ is actuated. The main control problems of the IWIP are periodic trajectory tracking [29], generation of limit cycle [30] [31] and stabilization where the position in which the pendulum is pointed upwards corresponds to the unstable equilibrium point. The dynamic model of IWIP can be obtained using the Euler-Lagrange method [32] [33] [24]. The equations of motion can be expressed by

$$
\left[\begin{array}{ll}
a+I_{W C} & I_{W C} \\
I_{W C} & I_{W C}
\end{array}\right]\left[\begin{array}{l}
\ddot{\theta}_{1} \\
\ddot{\theta}_{2}
\end{array}\right]-\left[\begin{array}{l}
b g \sin \left(\theta_{1}\right) \\
0
\end{array}\right]=\left[\begin{array}{l}
0 \\
u
\end{array}\right]
$$

where $\theta=\left[\theta_{1}, \theta_{2}\right]^{T}$, is the vector of generalized positions, $u$ is the torque generated by the actuator and acting on the inertia wheel. In the above model, the terms related to the friction in the pendulum joint are neglected. $a=M L^{2}+I_{P B}$ and $b=m l+M L$. The dynamic parameters of the system are $I_{P C}$ and $I_{P B}$ denote the rotational inertia of pendulum about pendulum center of mass (PC) and the rotational inertia of pendulum about its base (PB) respectively. $I_{W C}$ represents the rotational inertia of the

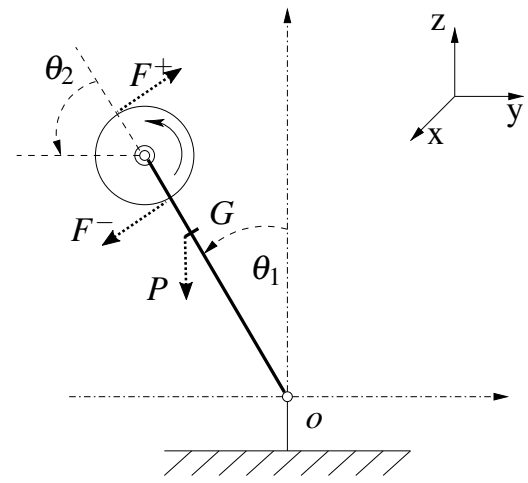

Fig. 1: Schematic view of the inertia wheel inverted pendulum.

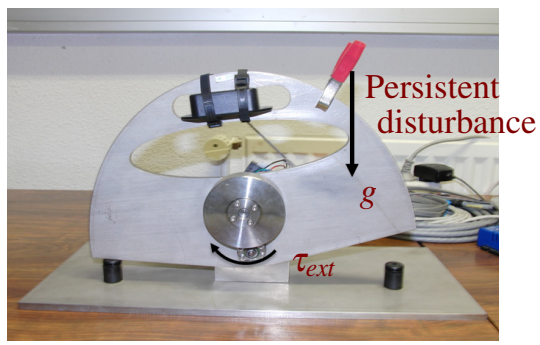

Fig. 2: Illustration of the external disturbance action applied on the pendulum body during experiments.

wheel about its center of mass (WC). $l$ denotes the Length from pendulum base ( $\mathrm{PB})$ to pendulum center of mass (PC), the length from pendulum base (PB) to wheel center of mass (WC) is denoted by $L, m$ and $M$ are mass of the pendulum and wheel respectively and $g$ is the constant of gravitational acceleration.

The first step is the transformation in $\mathrm{PCH}$ system. Using the following change of coordinates

$$
\begin{aligned}
& q_{1}=\theta_{1} \\
& q_{2}=\theta_{1}+\theta_{2} \\
& p_{1}=a \dot{q}_{1} \\
& p_{2}=I_{W C} \dot{q}_{2}
\end{aligned}
$$

We obtain the following simplified model with diagonal inertia matrix.

$$
\left(\begin{array}{ll}
a & 0 \\
0 & I_{W C}
\end{array}\right)\left(\begin{array}{l}
\ddot{q}_{1} \\
\ddot{q}_{2}
\end{array}\right)-\left(\begin{array}{l}
b g \sin \left(q_{1}\right) \\
0
\end{array}\right)=\left(\begin{array}{l}
-1 \\
1
\end{array}\right)_{(32)} u
$$

The obtained model is rewritten through Hamilton's equations of motion as

$$
\left(\begin{array}{c}
\dot{q}_{1} \\
\dot{q}_{2} \\
\dot{p}_{1} \\
\dot{p}_{2}
\end{array}\right)=\left(\begin{array}{c}
\frac{p_{1}}{a} \\
\frac{p_{2}}{I_{W C}} \\
b g \sin \left(q_{1}\right)-u \\
u
\end{array}\right)
$$

where $q=\left[q_{1}, q_{2}\right]^{T}$ and $p=\left[p_{1}, p_{2}\right]^{T}$ are the generalized positions and momenta respectively. 


\section{B. Application of IDA-PBC based controllers}

According to [8], the IDA-PBC control law can be described as

$$
\begin{aligned}
& u_{i d a}=\gamma_{1} \sin q_{1}+k_{p}\left(q_{2}+\gamma_{2} q_{1}\right)+k_{v} k_{2}\left(\dot{q}_{2}+\gamma_{2} \dot{q}_{1}\right) \\
& u_{i d a}=\gamma_{1} \sin q_{1}+K_{1} q_{1}+K_{2} q_{2}+K_{3} p_{1}+K_{4} p_{2}
\end{aligned}
$$

where $k_{p}>0$ is a proportional gain, $k_{v}>0$ is a damping injection gain, $\gamma_{1}>b, \gamma_{2}=\frac{a \gamma_{1}}{I_{W C}\left(\gamma_{1}-b\right)}$ and $k_{2}>0$.

$$
\begin{aligned}
K_{1} & =k_{p} \gamma_{2} \\
K_{2} & =k_{2} \\
K_{3} & =\frac{k_{v} k_{2} \gamma_{2}}{a} \\
K_{4} & =\frac{k_{v} k_{2}}{I_{W C}}
\end{aligned}
$$

According to [6], the MRA-IDA-PBC control law can be described as

$$
u_{M R A}=u_{i d a}+\Delta(x, t) \hat{z}
$$

where

$$
\begin{aligned}
\Delta(x, t) & =\left[p_{2}, p_{1}\right]^{T} \\
\hat{z} & =\left[\hat{K}_{3}, \hat{K}_{4}\right]
\end{aligned}
$$

The adaptation law is expressed as follows

$$
\dot{\hat{z}}=-K_{a} \Delta(x, t)^{T} G(x)^{T} \Delta_{p} H_{d}
$$

where $K_{a}$ is a diagonal positive definite matrix.

The PI-IDA-PBC control law expressed by Eq.(14)(17), while considering $\nabla V_{d}(q)=\left[\alpha\left[\cos \left(q_{1}\right)-1\right], 0\right]^{T}$ and $M_{d}$ a constant matrix described by

$$
M_{d}=\left(\begin{array}{ll}
a_{1} & a_{2} \\
a_{2} & a_{3}
\end{array}\right)
$$

with $a_{1}>0, a_{1} a_{3}>a_{2}^{2}$, and the input matrix $G$ is expressed by $G=[-1,1]^{T}$. The inertia matrix $M$ chosen for the (IWIP) is described as

$$
M=\left(\begin{array}{cc}
a & 0 \\
0 & I_{W C}
\end{array}\right)
$$

\section{REAL-TIME EXPERIMENTAL RESULTS}

In this section, we will compare experimentally the performance of IDA-PBC based controllers described in section 3. The control design parameters are $a=0.032$, $K_{a}=[0.025,0 ; 0,0.025], I_{W C}=0.000417, \hat{K}_{3}(0)=3, \gamma_{1}=$ $6.120, \hat{K}_{4}(0)=8, K_{1}=0.0471, \zeta(0)=0.12, K_{2}=0.0005$ , $K_{I}=0.18, K_{3}=16.4, K_{P}=3.42, K_{4}=3.4, K_{2}=1.07$, $\alpha=1.8934$.

Real-time experiments have been performed on the benchmark of the inertia wheel inverted pendulum system shown in Fig. 3. The position angle of the pendulum $\theta_{1}$ with respect to the vertical is measured by an incremental inclinometer FAS-G of micro Strain. The inertia wheel angular position $\theta_{2}$ is measured by an encoder integrated to the actuator (Maxon EC-powermax 30 DC motor) of the system.

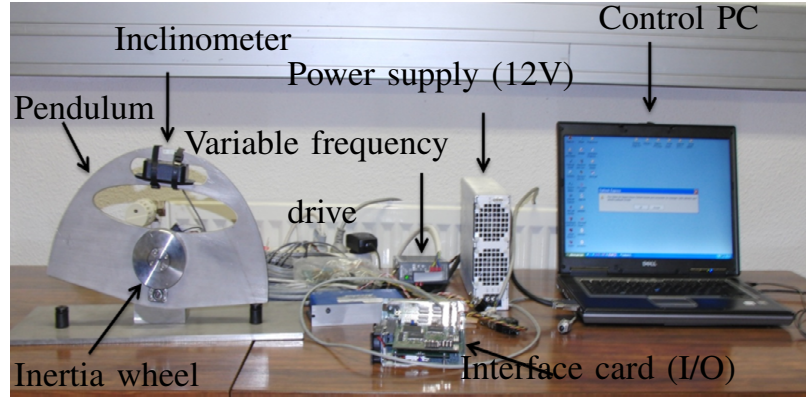

Fig. 3: View of the IWIP experimental testbed

Two experimental scenarios have been performed on the experimental testbed described above. In the first scenario, no external disturbances have been considered, while in this second one, an additional mass is attached to the body of the pendulum, introducing a persistent external disturbance acting on the passive joint of the pendulum.

1) Scenario 1: Stabilization in the Nominal case:

First, we applied the MRA-IDA-PBC controller. The proposed experiments were started from the initial condition $\left(q_{1}, q_{2}, p_{1}, p_{2}, \hat{K}_{3}, \hat{K}_{4}\right)^{T}=$ $\left(0.17,00,0, \hat{K}_{3}(0), \hat{K}_{4}(0)\right)^{T}$. Second, we applied the PI-IDA-PBC controller starting from the configuration $\left(q_{1}, q_{2}, p_{1}, p_{2}, \zeta\right)^{T}=(0.17,0,0,0, \zeta(0))^{T}$.
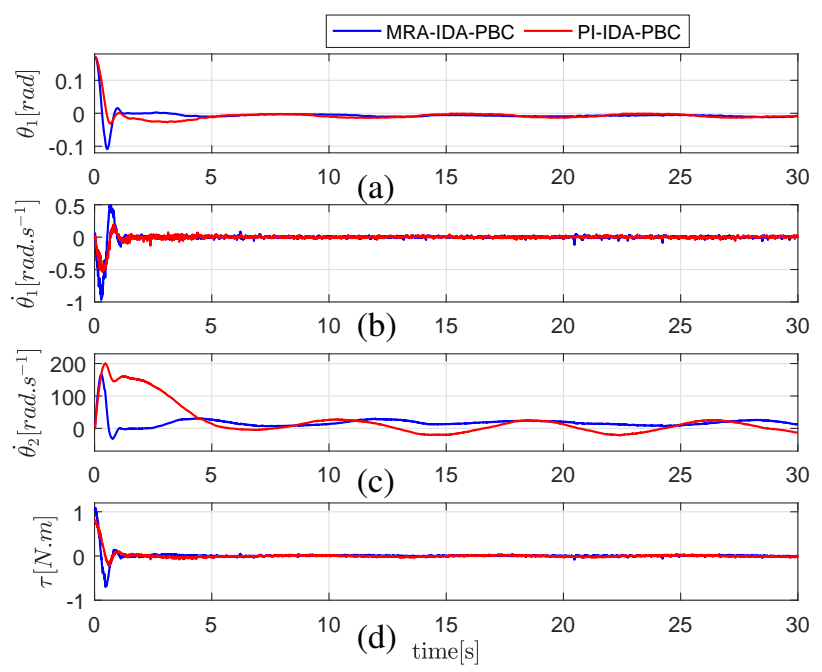

Fig. 4: Obtained experimental results for scenario 1: Nominal case. (a): Pendulum angular position, (b): pendulum angular velocity, (c): velocity of the inertia wheel, (d): The control input.

The obtained results in terms of angular positions and velocities are depicted in Fig. 4.(a,b,c). It can be clearly observed that the convergence with PI-IDA-PBC is better than with MRA-IDA-PBC controller. The evolution of the control input of the PI-IDA-PBC described in Fig. 4(d) converges faster to a steady state than the one of the MRA-IDA-PBC controller and presents also less oscillations. 
2020 17th International Multi-Conference on Systems, Signals \& Devices (SSD'20)
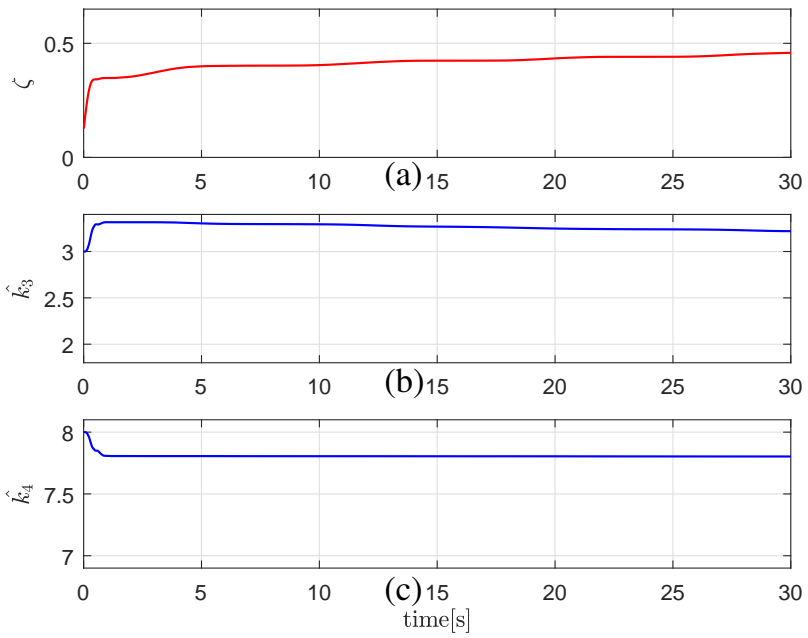

Fig. 5: Obtained experimental results for scenario 1: Nominal case.(a):The time evolution of $\zeta$, (b): The time evolution of $\hat{K}_{3}$, (c): The time evolution of $\hat{K}_{4}$.

The evolution of estimated gains $\zeta, \hat{K}_{3}$ and $\hat{K}_{4}$ for both PI-IDA and MRA-IDA are presented in Fig. 5 respectively. The figures describe the convergence of the estimated gain and PI parameters to their steady state values. The phase portrait depicted in Fig. 6, allows us to visually observe the trajectories of the dynamic system. Starting from the same initial condition, we can observe that the shortest path corresponds to the PI-IDA-PBC controller.

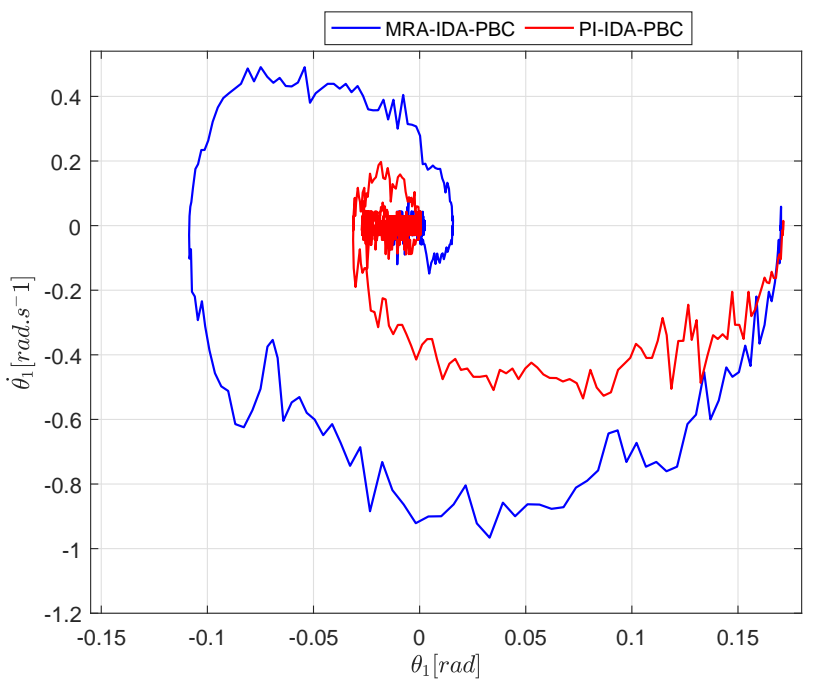

Fig. 6: Phase portrait $\left(\theta_{1}, \dot{\theta}_{1}\right)$ for scenario1.

In order to quantify the control performance of each controller in terms of convergence, let us consider the following criteria:

The root mean square of the tracking error (RMSE)

$$
R M S E=\left(\frac{1}{N} \sum_{i=1}^{N}\left(e_{\theta_{1}}(i)\right)^{2}\right)^{\frac{1}{2}}
$$

TABLE I: Quantification of the performance through different evaluation criteria for scenario 1

\begin{tabular}{|c|c|c|}
\hline Criteria & \multicolumn{2}{|c|}{ Scenario1 } \\
\hline & MRA-IDA-PBC & PI-IDA-PBC \\
\cline { 2 - 3 } RMSE [rad] & 0.0035 & 0.0012 \\
ISE & 0.0098 & 0.0076 \\
IAE & 0.1951 & 0.1885 \\
ITAE & 0.0142 & 0.0049 \\
$E_{\tau}[$ N. $m]$ & 124.6523 & 89.2769 \\
\hline
\end{tabular}

TABLE II: Quantification of the performance through different evaluation criteria for scenario 2

\begin{tabular}{|c|c|c|}
\hline Criteria & \multicolumn{2}{|c|}{ Scenario2 } \\
\hline \multirow{2}{*}{ RMSE [rad] } & MRA-IDA-PBC & PI-IDA-PBC \\
\cline { 2 - 3 } ISE & 0.0199 & 0.0187 \\
IAE & 0.0198 & 0.0171 \\
ITAE & 0.6656 & 0.5822 \\
$E_{\tau}[N . m]$ & 0.2228 & 0.1655 \\
& 126.199 & 112.202 \\
\hline
\end{tabular}

The Integral Square Error (ISE)

$$
I S E=\int e_{\theta_{1}}^{2} d t
$$

Inegral Absolute Error (IAE)

$$
I A E=\int\left|e_{\theta_{1}}\right| d t
$$

Integral Time-weighted Absolute Error (ITAE)

$$
I T A E=\int t\left|e_{\theta_{1}}\right| d t
$$

where $N$ is the number of the recorded samples, and $e_{\theta_{1}}$ denotes the tracking error of the unactuated joint $\theta_{1}$.

To compare the obtained performance in terms of energy consumption, the following input-torques based criterion can be

$$
E_{\tau}=\sum_{i=1}^{N}\left|\tau_{\theta_{2}}(i)\right|
$$

where $\tau_{\theta_{2}}=u$ is the torque generated by the actuator and acting on the inertia wheel.

Based on the proposed performance-evaluation criteria, the obtained results are illustrated in TABLE I. Note that, the smaller value of performance criteria indicates the best performance. It can be clearly concluded from those results that the MRA-IDA-PBC provides satisfactory results, but the PI-IDA-PBC has significantly the best performance in terms of convergence and energy consumption.

2) Scenario 2: Persistent external disturbance rejection: The disturbance illustrated in Fig. 2 is persistent and applied permanently to the inverted pendulum as an additional mass attached to the pendulum body. It can be representing an external force $F_{d i s}$ applied on the inverted pendulum. This force generate a torque $\tau_{e x t}$ around the pendulum pivot passive joint. In this section, the ability of the two implemented controllers to reject this external disturbance is studied. 
The obtained experimental results of the second scenario are displayed in Fig. $7(\mathrm{a}, \mathrm{b}, \mathrm{c})$. It presents the evolution of the angular position on the pendulum $\theta_{1}$, the angular velocity of the pendulum $\dot{\theta}_{1}$ and the velocity of the inertia wheel $\dot{\theta}_{2}$, versus time. Fig. 7(d) depicts the evolution of the control inputs. The two controllers reject the introduced persistent disturbance and keep the system close to its unstable equilibrium point $\left(\theta_{1}, \dot{\theta}_{1}\right)=(0,0)$. In the case of PI-IDA-PBC controller, the rotation of the actuator is permanent and the disturbance does not significantly affect the evolution of the angular velocity of the inertia wheel. However, in the case of MRAIDA-PBC controller, we can observe the effect of the persistent disturbance on the behaviour of the angular velocity of the inertia wheel as a shift in the velocity curve.

The experimental results and the evaluation based on the different performance criteria in TABLE II show that the PI-IDA-PBC ensure a better convergence with respect to the MRA-IDA-PBC controller.
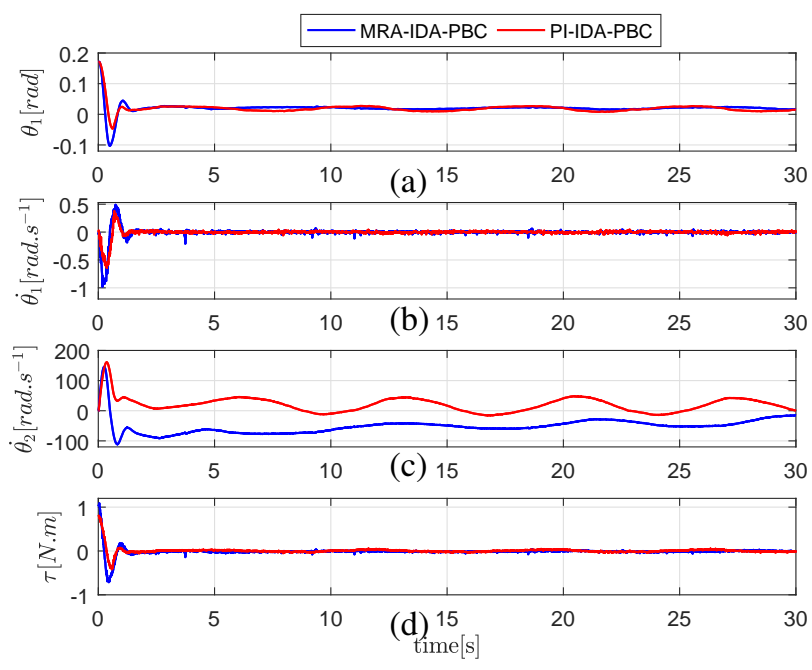

Fig. 7: Obtained experimental results for scenario 2: Persistent disturbance rejection. (a): Pendulum angular position, (b): Pendulum angular velocity, (c): Velocity of the inertia wheel, (d): The control input.

The generated control input torques for both controllers are illustrated in Fig. 7(d). Both controllers apply a DC voltage to the motor, which makes it rotate permanently to compensate the disturbing torque and thus allow the rejection of the external disturbance. Using the input-torques based criterion summarized in TABLE II, we conclude that the PI-IDA-PBC is better in terms of energy consumption as well as tracking performance. The real-time evolution of the controller state $\zeta$ of PIIDA-PBC and the parameters of the adaptive controller $\left(\hat{K}_{3}, \hat{K}_{4}\right)$ are displayed in Fig. 8(a, b, c) respectively. We can observe the convergence of these parameters to their steady-state values.
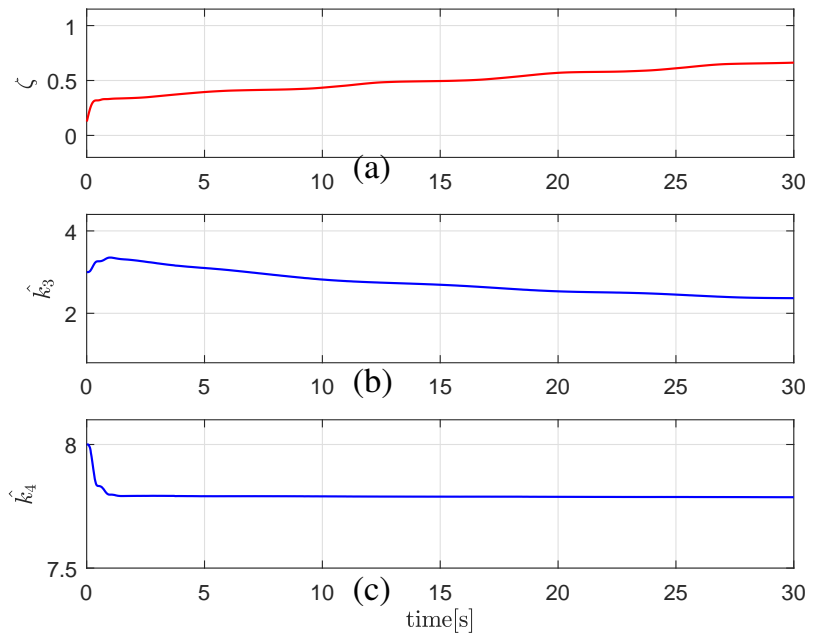

Fig. 8: Obtained experimental results for scenario 2: Persistent disturbance rejection.(a):The evolution of $\zeta$, (b): The evolution of $\hat{K}_{3}$, (c): The evolution of $\hat{K}_{4}$ versus time.

\section{CONCLUSION AND FUTURE WORK}

This paper presented an experimental comparative study of the behaviour of two controllers based on IDAPBC namely, PI-IDA-PBC and MRA-IDA-PBC controllers. The first one is an adaptive control scheme combined with the IDA-PBC design methodology. The second one is a an outer-loop controller added to the interconnection and damping assignment passivity-based control. Both controllers were primarily designed to improve the robustness of the standard IDA-PBC for underactuated mechanical system.

The comparative study was conducted experimentally for the case of the inertia wheel inverted pendulum (IWIP). The objective was to stabilize the inertia wheel inverted pendulum around its unstable equilibrium point, and to maintain it in this state despite the presence of external disturbances. Two experimental scenarios were considered with implementation issues. The first one concerned the control of the nominal system without perturbations, while in the second one, the system was subject to an external persistent perturbation.

Based on the obtained experimental results and different performance-evaluation criteria, the PI-IDA-PBC has significant performance in term of convergence and energy consumption compared to the MRA-IDA-PBC controller. In future work, various possible extensions of this work can be investigated. At first, we can combine the adaptation law with the PI to estimate the proportional and integral gains. Furthermore, discussions can be investigated about the generalization of this study to the case of other classes of underactuated mechanical systems. Finally, an automatic optimal tuning of the control design parameter of both controllers can also be investigated. 


\section{REFERENCES}

[1] S. Andary, A. Chemori, and S. Krut, "Control of the underactuated inertia wheel inverted pendulum for stable limit cycle generation," Advanced Robotics, vol. 23, no. 15, pp. 1999-2014, 2009.

[2] A. Choukchou-Braham, B. Cherki, M. Djemai, and K. Busawon, Analysis and Control of Underactuated Mechanical Systems. Switzerland: Springer International Publishing, 2014.

[3] M. Spong, "Underactuated mechanical systems:control problems in robotics and automation." Berlin, Heidelberg, pp. 135-150, 1998, lecture Notes in Control and Information Sciences.

[4] S. Krafes, Z. Chalh, and A. Saka, "A review on the control of second order underactuated mechanical systems," Complexity, vol. 2018, p. 17, 2018.

[5] A. J. Van der Schaft, "Port-controlled hamiltonian systems: Towards a theory for control and design of nonlinear physical systems," Journal of the Society of Instrument and Control Engineers, vol. 39, no. 2, pp. 91-98, 2000.

[6] N. Khraief Haddad, A. Chemori, and S. Belghith, "Robustness enhancement of ida-pbc controller in stabilising the inertia wheel inverted pendulum: theory and real-time experiments," International Journal of Control, vol. 91, no. 12, pp. 2657-2672, 2018.

[7] M. Ryalat and D. S. Laila, "A robust ida-pbc approach for handling uncertainties in underactuated mechanical systems," IEEE Transactions on Automatic Control, vol. 63, no. 10, pp. 34953502 , Oct 2018.

[8] R. Ortega, M. W. Spong, F. Gomez-Estern, and G. Blankenstein, "Stabilization of a class of underactuated mechanical systems via interconnection and damping assignment," IEEE Transactions on Automatic Control, vol. 47, no. 8, pp. 1218-1233, Aug 2002

[9] H. Rodriguez, R. Ortega, G. Escobar, and N. Barabanov, "A robustly stable output feedback saturated controller for the boost dc-to-dc converter," Systems and Control Letters, vol. 40, no. 1, pp. $1-8,2000$.

[10] H. Gonzalez, M. Duarte-Mermoud, I. Pelissier, J. Travieso, and R. Ortega, "A novel induction motor control scheme using idapbc," Journal of Control Theory and Applications, vol. 6, pp. 59-68, 022008.

[11] H. Ramírez, D. Sbarbaro, and R. Ortega, "On the control of non-linear processes: An ida-pbc approach," Journal of Process Control, vol. 19, no. 3, pp. $405-414,2009$.

[12] S. Aoues, D. Matignon, and D. Alazard, "Control of a flexible spacecraft using discrete ida-pbc design," IFAC-PapersOnLine, vol. 48, no. 13 , pp. 188 - 193, 2015, 5th IFAC Workshop on Lagrangian and Hamiltonian Methods for Nonlinear Control LHMNC 2015

[13] F. Gomez-Estern and A. J. Van der Schaft, "Physical damping in ida-pbc controlled underactuated mechanical systems," European journal of control, vol. 10, no. 5, pp. 451-468, 12 2004, special Issue on Hamiltonian and Lagrangian Methods for Nonlinear Control.

[14] G. Viola, R. Ortega, R. Banavar, J. A. Acosta, and A. Astolfi, “Total energy shaping control of mechanical systems: Simplifying the matching equations via coordinate changes," IEEE Transactions on Automatic Control, vol. 52, no. 6, pp. 1093-1099, June 2007.

[15] M. Ryalat and D. S. Laila, "Ida-pbc for a class of underactuated mechanical systems with application to a rotary inverted pendulum," in 52nd IEEE Conference on Decision and Control, Florence, Italy, Florence, Italy, Dec 2013, pp. 5240-5245.

[16] V. Santibanez, R. Kelly, and J. Sandoval, "Control of the inertia wheel pendulum by bounded torques," in Proceedings of the 44th IEEE Conference on Decision and Control, Seville, Spain, Seville, Spain, Dec 2005, pp. 8266-8270.

[17] F. Tiefensee, S. Monaco, and D. Normand-Cyrot, "Ida-pbc under sampling for port-controlled hamiltonian systems," in Proceedings of the 2010 American Control Conference, Baltimore, MD, USA, Baltimore, MD, USA, June 2010, pp. 1811-1816.

[18] M. Ryalat, D. S. Laila, and M. M. Torbati, "Integral ida-pbc and pid-like control for port-controlled hamiltonian systems," in 2015 American Control Conference (ACC), Chicago, IL, USA, Chicago, IL, USA, July 2015, pp. 5365-5370.

[19] A. Donaire, J. G. Romero, R. Ortega, B. Siciliano, and M. Crespo, "Robust ida-pbc for underactuated mechanical systems subject to matched disturbances," International Journal of Robust and Nonlinear Control, vol. 27, no. 6, pp. 1000-1016, 2017.
[20] N. Khraief Haddad, A. Chemori, J. J. Pena, and S. Belghith, "Stabilization of inertia wheel inverted pendulum by model reference adaptive ida-pbc: From simulation to real-time experiments," in 2015 3rd International Conference on Control, Engineering Information Technology (CEIT), Tlemcen, Algeria, Tlemcen, Algeria, May 2015, pp. 1-6.

[21] N. Khraief Haddad, A. Chemori, and S. Belghith, "External disturbance rejection in ida-pbc controller for underactuated mechanical systems: From theory to real time experiments," in 2014 IEEE Conference on Control Applications (CCA), Juan Les Antibes, France, Juan Les Antibes, France, Oct 2014, pp. 17471752.

[22] M. W. Spong, P. Corke, and R. Lozano, "Nonlinear control of the reaction wheel pendulum," Automatica, vol. 37, no. 11, pp. 1845 $-1851,2001$.

[23] L. Freidovich, P. X. La Hera, U. Mettin, A. Robertsson, A. S Shiriaev, and R. Johansson, "Shaping stable periodic motions of inertia wheel pendulum: Theory and experiment," Asian Journal of Control, vol. 11, no. 5, pp. 548 - 556, 092009.

[24] S. Andary, A. Chemori, M. Benoit, and J. Sallantin, "A dual model-free control of underactuated mechanical systems, application to the inertia wheel inverted pendulum," in 2012 American Control Conference (ACC), Montreal, QC, Canada, 2012, pp. 1029-1034.

[25] C. Aguilar-Avelar, R. Rodríguez-Calderon, S. Puga-Guzmán, and J. Moreno-Valenzuela, "Effects of nonlinear friction compensation in the inertia wheel pendulum," Journal of Mechanical Science and Technology, vol. 31, no. 9, pp. 4425-4433, Sep 2017.

[26] A. Hfaiedh, A. Chemori, and A. Abdelkrim, "Rise controller for class I underactuated mechanical systems: Design and real-time experiments," in The 3rd International Conference on Electromechanical Engineering (ICEE'2018), Skikda, Algeria, 2018.

[27] J. Ghommam and A. Chemori, "Adaptive rbfnn finite-time control of normal forms for underactuated mechanical systems," Nonlinear Dyn, vol. 90, no. 1, p. 301-315, 2017.

[28] S. Andary and A. Chemori, "A dual model-free control of nonminimum phase systems for generation of stable limit cycles," in 2011 50th IEEE Conference on Decision and Control and European Control Conference, Orlando, FL, USA, 2011, pp. 1387-1392.

[29] C. Zayane-Aissa, T. Laleg-Kirati, and A. Chemori, "Control of a perturbed under-actuated mechanical system," in 2015 IEEE Conference on Control Applications (CCA), Sydney, Australia, 2015, pp. 294-299.

[30] H. Gritli, K. Nahla, A. Chemori, and S. Belghith, "Self-generated limit cycle tracking of the underactuated inertia wheel inverted pendulum under ida-pbc," Nonlinear Dynamics, vol. 29, no. 3, 052017.

[31] N. Haddad, S. Belghith, H. Gritli, and A. Chemori, "From hopf bifurcation to limit cycles control in underactuated mechanical systems," International Journal of Bifurcation and Chaos, vol. 27, no. 7, p. 1750104, 062017.

[32] S. Andary, A. Chemori, and S. Krut, "Stable limit cycle generation for underactuated mechanical systems, application: Inertia wheel inverted pendulum," in 2008 IEEE/RSJ International Conference on Intelligent Robots and Systems, Nice, France, 2008, pp. 526-531.

[33] $\_$, "Estimation-based disturbance rejection in control for limit cycle generation on inertia wheel inverted pendulum testbed," in 2009 IEEE/RSJ International Conference on Intelligent Robots and Systems, St Louis, USA, 2009, pp. 1302-1307. 